\title{
(6) OPEN ACCESS \\ Economic evaluation of a hospital-initiated intervention for smokers with chronic disease, in Ontario, Canada
}

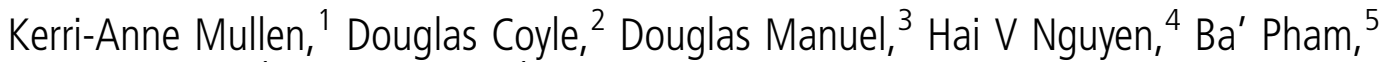 \\ Andrew L Pipe, ${ }^{1}$ Robert D Reid ${ }^{1}$
}

${ }^{1}$ Division of Prevention and Rehabilitation, University of Ottawa Heart Institute, Ottawa, Ontario, Canada ${ }^{2}$ Department of Epidemiology and Community Medicine, University of Ottawa, Ottawa, Ontario, Canada ${ }^{3}$ Clinical Epidemiology, Ottawa Hospital Research Institute, Ottawa, Ontario, Canada ${ }^{4}$ Program in Health Services and Systems Research, DukeNUS Graduate Medical School, Singapore, Singapore 5 Institute of Health Policy, Management and Evaluation, University of Toronto, Toronto, Ontario, Canada

\section{Correspondence to} Kerri-Anne Mullen, Division of Prevention and Rehabilitation, University of Ottawa Heart Institute, Room H2353, 40 Ruskin Street, Ottawa, Ontario, Canada K1Y4W7; kmullen@ottawaheart.ca

Received 5 December 2013 Revised 21 May 2014 Accepted 23 May 2014 Published Online First 16 June 2014

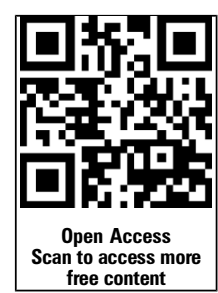

CrossMark

\section{To cite: Mullen $\mathrm{KA}$,}

Coyle D, Manuel $D$, et al.

Tob Control 2015;24

489-496.

\begin{abstract}
Introduction Cigarette smoking causes many chronic diseases that are costly and result in frequent hospitalisation. Hospital-initiated smoking cessation interventions increase the likelihood that patients will become smoke-free. We modelled the cost-effectiveness of the Ottawa Model for Smoking Cessation (OMSC), an intervention that includes in-hospital counselling, pharmacotherapy and posthospital follow-up, compared to usual care among smokers hospitalised with acute myocardial infarction (AMI), unstable angina (UA), heart failure (HF), and chronic obstructive pulmonary disease (COPD).
\end{abstract}

Methods We completed a cost-effectiveness analysis based on a decision-analytic model to assess smokers hospitalised in Ontario, Canada for AMI, UA, HF, and COPD, their risk of continuing to smoke and the effects of quitting on re-hospitalisation and mortality over a 1-year period. We calculated short-term and long-term cost-effectiveness ratios. Our primary outcome was 1-year cost per quality-adjusted life year (QALY) gained. Results From the hospital payer's perspective, delivery of the OMSC can be considered cost effective with 1-year cost per QALY gained of \$C1386, and lifetime cost per QALY gained of $\$ C 68$. In the first year, we calculated that provision of the OMSC to 15326 smokers would generate 4689 quitters, and would prevent 116 rehospitalisations, 923 hospital days, and 119 deaths. Results were robust within numerous sensitivity analyses.

Discussion The OMSC appears to be cost-effective from the hospital payer perspective. Important consideration is the relatively low intervention cost compared to the reduction in costs related to readmissions for illnesses associated with continued smoking.

\section{INTRODUCTION}

Smoking-related illnesses are principal drivers of healthcare spending; they are estimated to contribute up to $15 \%$ of healthcare expenditures in developed nations. ${ }^{1}$ Many of the chronic diseases caused by cigarette smoking result in frequent hospitalisation making the hospital an ideal setting to initiate cessation treatment. ${ }^{2}$ Rigotti and colleagues recently updated a review and meta-analysis of studies examining the efficacy of hospital-initiated smoking cessation interventions. The interventions included in the review were offered by hospital staff (eg, physicians, nurses, or other allied health professionals), and could involve the provision of advice, intensive counselling, pharmacotherapy and follow-up contact after hospital discharge. The authors concluded that smoking cessation support that began in hospital and continued for at least 1 month after discharge significantly increased the likelihood of patients being smoke-free in the long term (risk ratio $=1.37,95 \%$ CI 1.27 to $1.48 ; 25$ trials), and that strategies that included counselling and pharmacotherapy were more efficacious than just counselling alone (relative risks $(\mathrm{RR})=1.54$, 95\% CI 1.34 to 1.79 , six trials). ${ }^{3}$

One of the studies included in the Rigotti review was by researchers at the University of Ottawa Heart Institute, of an intervention that is now known as the Ottawa Model for Smoking Cessation (OMSC). ${ }^{4}$ The OMSC is a systematic approach to the identification, treatment and follow-up of smokers that is embedded within hospital management systems using organisational change strategies. The OMSC has been found to significantly increase long-term cessation rates by an absolute $15 \%$ (from $29 \%$ to $44 \%$ ) in cardiac patients, and by $11 \%$ (from $18 \%$ to $29 \%$ ) in general hospital populations. ${ }^{45}$

Patients who quit smoking during hospitalisation are less likely to be rehospitalised or to die during follow-up. ${ }^{67}$ Despite such evidence, most hospitals fail to deliver cessation interventions due, in part, to concerns about the perceived costs of such programmes. Economic evaluations are becoming increasingly popular in helping healthcare administrators choose whether or not to fund interventions, and where to devote resources. ${ }^{8}$ Cost-effectiveness analysis (CEA) is a type of economic evaluation that examines the consequences or gains of an intervention compared to an alternative. The results of CEA are expressed as costeffectiveness ratios. For example, outcomes of CEA for a smoking cessation programme might include the cost per number of people who quit smoking, cost per number of hospital-days prevented by the programme, or cost per number of years of life gained by the programme. Cost-utility analysis (CUA) is a form of CEA that examines the cost of an intervention relative to the benefit it produces in terms of the number of years of life gained, combined with the quality of those years lived. The quality-adjusted life-year (QALY) is the most common outcome measured in a CUA; it is a costeffectiveness ratio that takes into account quantity and health-related quality of life. ${ }^{9}$

The purpose of this study was to determine, from the hospital payer's perspective, the short-term (1 year) and long-term (lifetime) cost-effectiveness 
of the OMSC intervention, as compared to a usual care condition, among smokers hospitalised with acute myocardial infarction (AMI), unstable angina (UA), heart failure (HF), or chronic obstructive pulmonary disease (COPD). This perspective and these four diagnoses were selected for our analysis due to the particular burden of these tobacco-related diseases on hospitalisation and rehospitalisation, and the availability of data.

\section{METHODS}

\section{Setting}

Our study examined patients hospitalised in Ontario, a Canadian province with a population of approximately 12.8 million. The smoking prevalence among Ontarians aged 15 years and older was $15.4 \%$ in $2009 .^{10}$ The smoking prevalence among Canadian hospital populations is higher, at $20 \%{ }^{11}$; consequently, on a given day in Ontario, approximately 6400 of the province's 32000 hospital beds are occupied by current smokers. Healthcare is publicly funded through the Ontario Health Insurance Plan, where medically necessary services including primary care and specialty care, hospital stays, diagnostic investigations and surgical procedures are available at no cost to residents. There are 211 hospital sites in Ontario that recorded an estimated 987757 acute care inpatient admissions in $2009 .^{12}$

\section{Population}

Our analysis included smokers hospitalised for one of the four selected diagnoses. In 2009, there were 20 503, 5370, 16339 and 17585 unique admissions to Ontario hospitals for AMI, UA, HF and COPD, respectively, for a total of 59797, as reported by the Canadian Institute for Health Information's (CIHI) Discharge Abstract Database (DAD). ${ }^{13}$ We combined data from a previously published quasi-experimental, before and after cohort study of the OMSC conducted at nine Ontario hospitals, ${ }^{5}$ with data from similar evaluations that took place at an additional 19 Ontario hospitals, in order to estimate the number of these unique admissions that involved smokers, and to estimate programme quit rates. Our study dataset included patients $(\mathrm{n}=3269)$ aged 18 years or older who were hospitalised for either AMI $(n=750)$, UA $(n=705)$, HF $(n=855)$, or COPD $(n=956)$. Smokers were defined as anyone who reported having smoked daily ( $\geq 1$ cigarette per day) in the 6 months leading up to their hospitalisation. The overall smoking prevalence was $25.4 \%$; disease-specific smoking rates are displayed in table 1. Applying the disease-specific smoking prevalence to the number of unique admissions, we estimated that 15326 smokers were hospitalised in 2009 for AMI $(\mathrm{n}=5194), \quad \mathrm{UA} \quad(\mathrm{n}=1424), \quad \mathrm{HF} \quad(\mathrm{n}=2804)$, and COPD $(\mathrm{n}=5904)$.

Table 1 Data elements included in the base case analysis (\$ in \$C)

\begin{tabular}{|c|c|c|c|c|c|}
\hline & AMI & UA & HF & COPD & Sources \\
\hline Smoking prevalence rates $(95 \% \mathrm{Cl})$ & $0.25(0.22$ to 0.29$)$ & $0.27(0.23$ to 0.30$)$ & $0.17(0.14$ to 0.21$)$ & 0.34 (0.29 to 0.38$)$ & 5 \\
\hline \multicolumn{6}{|l|}{ Age, mean (SD) } \\
\hline Usual care & $56.9(10.2)$ & $58.0(10.5)$ & $60.8(13.0)$ & $62.3(15.4)$ & 5 \\
\hline OMSC & $57.4(13.1)$ & $56.6(12.9)$ & $61.4(10.4)$ & $65.9(10.6)$ & 5 \\
\hline \multicolumn{6}{|l|}{ 12-month smoking abstinence rates $(95 \% \mathrm{Cl})$} \\
\hline Usual care & $0.22(0.15$ to 0.30$)$ & $0.20(0.13$ to 0.30$)$ & $0.17(0.10$ to 0.27$)$ & $0.13(0.08$ to 0.21$)$ & 5 \\
\hline OMSC & $0.38(0.28$ to 0.51$)$ & $0.35(0.25$ to 0.48$)$ & $0.28(0.20$ to 0.39$)$ & $0.24(0.19$ to 0.30$)$ & \\
\hline \multicolumn{6}{|l|}{ Risk of one rehospitalisation in first year $(95 \% \mathrm{Cl})$} \\
\hline Continue to smoke & $0.05(0.03$ to 0.06$)$ & $0.10(0.09$ to 0.12$)$ & 0.17 (0.16 to 0.19$)$ & $0.18(0.17$ to 0.20$)$ & $717-20$ \\
\hline Quit smoking & $0.04(0.03$ to 0.05$)$ & $0.08(0.07$ to 0.10$)$ & $0.14(0.13$ to 0.16$)$ & $0.15(0.14$ to 0.16$)$ & \\
\hline \multicolumn{6}{|l|}{ Risk of two or more rehospitalisations in first year $(95 \% \mathrm{Cl})$} \\
\hline Continue to smoke & $0.03(0.02$ to 0.04$)$ & $0.03(0.02$ to 0.05$)$ & $0.09(0.07$ to 0.11$)$ & $0.14(0.12$ to 0.15$)$ & $717-20$ \\
\hline Quit smoking & $0.02(0.01$ to 0.04$)$ & $0.03(0.01$ to 0.04$)$ & $0.08(0.06$ to 0.09$)$ & $0.11(0.10$ to 0.13$)$ & \\
\hline Rehospitalisation cost, mean (SD) & $\$ 9559$ (\$14 539) & $\$ 5950(\$ 6932)$ & $\$ 10029$ (\$16 696) & $\$ 8808(\$ 16795)$ & 21 \\
\hline Length of stay of rehospitalisation (days), mean (SD) & $5.6(9.8)$ & $4.1(4.9)$ & $9.7(12.8)$ & $8.4(14.5)$ & 21 \\
\hline \multicolumn{6}{|l|}{ Risk of death in first year $(95 \% \mathrm{Cl})$} \\
\hline Continue to smoke & $0.14(0.13$ to 0.15$)$ & $0.09(0.08$ to 0.10$)$ & $0.48(0.47$ to 0.49$)$ & $0.09(0.09$ to 0.09$)$ & $192022-25$ \\
\hline Quit smoking & $0.09(0.08$ to 0.10$)$ & $0.06(0.05$ to 0.07$)$ & 0.31 (0.30 to 0.31 ) & 0.06 (0.06 to 0.06$)$ & \\
\hline \multicolumn{6}{|l|}{ Utility scores } \\
\hline Continue to smoke & 0.53 & 0.52 & 0.48 & 0.50 & 2627 \\
\hline Quit smoking & 0.55 & 0.54 & 0.50 & 0.52 & \\
\hline \multicolumn{6}{|l|}{ Life expectancy, mean number of years remaining } \\
\hline Continue to smoke & 7.58 & 7.42 & 5.19 & 7.75 & $28-30$ \\
\hline Quit smoking & 8.76 & 8.64 & 6.07 & 9.01 & \\
\hline \multicolumn{6}{|l|}{ Intervention costs (per patient) } \\
\hline Personnel costs to complete in-hospital consultation & $\$ 20.63$ & $\$ 20.63$ & $\$ 20.63$ & $\$ 20.63$ & \\
\hline In-hospital pharmacotherapy & $\$ 13.95^{*}$ & $\$ 9.23^{*}$ & $\$ 21.60^{*}$ & $\$ 21.25^{*}$ & \\
\hline Automated follow-up system fee & $\$ 10.30$ & $\$ 10.30$ & $\$ 10.30$ & $\$ 10.30$ & \\
\hline Personnel costs to complete follow-up counselling calls & $\$ 27.24$ & $\$ 27.24$ & $\$ 27.24$ & $\$ 27.24$ & \\
\hline Total intervention cost & $\$ 71.50$ & $\$ 66.98$ & $\$ 78.81$ & $\$ 76.44$ & \\
\hline
\end{tabular}




\section{Intervention}

The OMSC was selected as the intervention in our model for several reasons. It is the most widely implemented hospital-initiated smoking cessation intervention in Canada. As of 2014, it had been implemented in approximately 100 (14\%) Canadian hospitals. It features a large programme database where data were available to generate some of our model's data elements, broken down by disease group. A Canadian intervention was preferred as our estimates of cost and risk of rehospitalisation were derived from Canadian sources.

As part of the OMSC intervention, the following takes places with each admitted smoker: (1) a 10-30 min consultation is completed by a nurse or another healthcare professional (eg, respiratory therapist) at the bedside, employing a standardised consultation and assessment form. The form gathers information on smoking history and readiness to quit. It guides the health professional in the selection and ordering of quit smoking medication, in giving practical advice, and registering the patient in the follow-up support programme; (2) pharmacotherapy (primarily nicotine replacement therapy-NRT) is offered to the patient for the duration of their hospital admission and ordered through the hospital pharmacy using standard medication order forms; (3) guidance regarding the use of smoking cessation pharmacotherapy following hospital discharge is provided; (4) a patient education booklet with information for those thinking about quitting, preparing to quit, ready to quit, or wanting to stay smoke-free is provided; (5) enrolment in a telephone follow-up system for 6 months after discharge is offered. The telephone follow-up is conducted using an automated system that places eight programmed calls to smokers on days 3,14, 30, 60, 90, 120, 150 and 180 after their discharge date. Patients respond to automated questions about their smoking status and their confidence in remaining smoke-free. Their responses serve as a triage tool, and the system flags those who have relapsed to smoking or who may have low confidence in remaining smoke-free. Nurse counsellors who specialise in smoking cessation treatment and relapse prevention monitor the system and call patients who are flagged as experiencing difficulty. The OMSC protocol and follow-up system have been previously described in greater detail. ${ }^{4} 514$

\section{Usual care}

A number of guidelines exist recommending the integration of tobacco cessation interventions within clinical practices in Canada ${ }^{15}{ }^{16}$; however, the large majority of hospitals in Canada do not have in place smoking cessation protocols or programmes. Therefore, the most common alternative to our intervention is usual care, which may consist of the recording of smoking status on the patient chart and possibly the provision of a patient education booklet.

\section{Model framework}

A decision analytic model was developed using an Excel spreadsheet (Microsoft Corporation, USA). It included smokers hospitalised with AMI, UA, HF and COPD (15 326 in each the usual care and OMSC branches), their RRs of continuing to smoke, and the transitions to various states relating to health outcomes among those who continued to smoke versus those who had quit over the 1-year period (figure 1). The following 1-year health states were included: no adverse outcomes, one rehospitalisation, two or more rehospitalisations, or death.

\section{Data elements}

The data elements used in our model's base case analysis are listed in table 1 and their sources identified.

\section{Smoking abstinence rates}

Smoking abstinence rates were obtained from our study dataset. Self-reported 6-month continuous quit rates (ie, patient responded 'no' when asked 'have you used any form of tobacco in the past 6 months?') had been collected on a sample of smokers who received either usual care $(n=369)$ or OMSC $(n=439)$. They were calculated using intention-to-treat analysis (ie, we assumed that those who were lost to follow-up were still smoking) and were broken down by diagnosis. A 5\% relapse rate was applied to our 6-month quit rates in order to estimate 1-year quitting in our model based on a 2008 meta-analysis of relapse by Hughes et al. ${ }^{31}$ Overall, the 12 -month quit rate $(95 \%$ $\mathrm{CI})$ was $17.9 \%(14.0 \%$ to $21.8 \%)$ for usual care and $28.5 \%$ (24.3\% to $32.7 \%$ ) for the OMSC intervention. Disease-specific rates are presented in table 1 .

\section{Rehospitalisation}

We obtained values for the risk of being readmitted to hospital one or two or more times in the year following the index hospitalisation for each diagnosis using data provided by CIHI. These values were assigned to patients who had quit smoking. For continued smokers, the risk values were multiplied by the following $\mathrm{RR}$ of being rehospitalised: 1.21 for $\mathrm{AMI}$ or $\mathrm{UA}^{7}$; 1.20 for

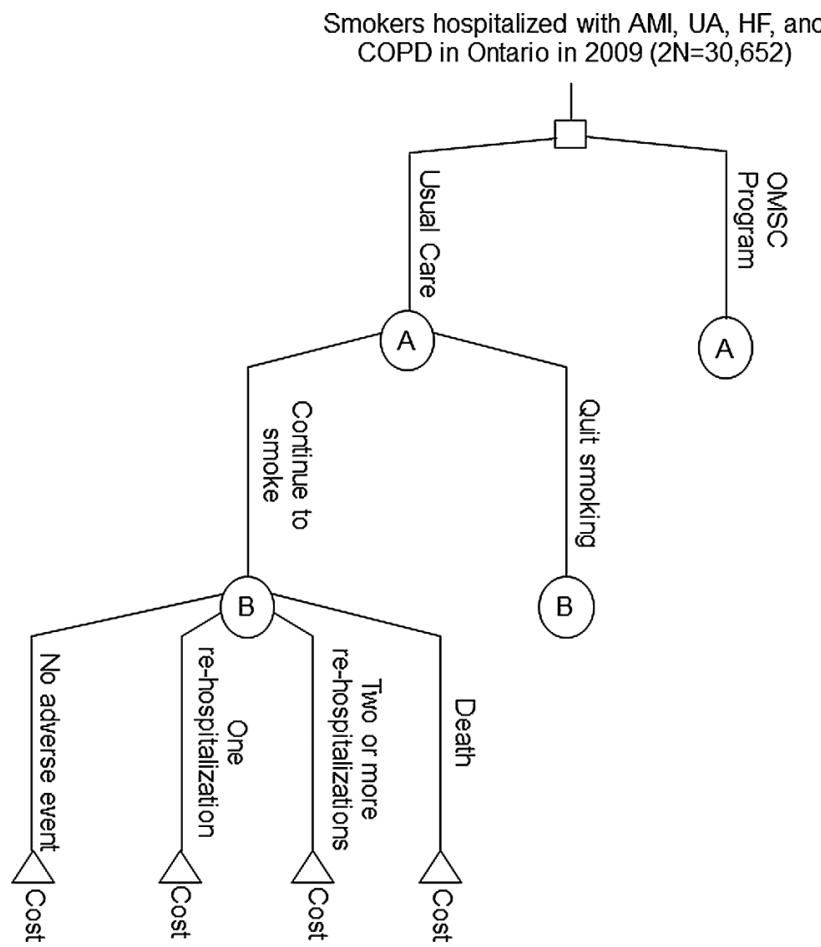

Figure 1 Decision analytic model. The square represents a decision node. In this case we are evaluating the difference between hospitalised smokers with $\mathrm{AMI}, \mathrm{UA}, \mathrm{HF}$, or COPD receiving either usual care or the OMSC programme. Circles A and B are chance nodes and indicate where probabilities of two or more events occur (the events are identical for each group but are only displayed for usual care). The triangles indicate terminal nodes and are the end points we wish to evaluate. AMI, acute myocardial infarction; COPD, chronic obstructive pulmonary diseases; HF, heart failure; OMSC, Ottawa model for smoking cessation; UA, unstable angina pectoris. 
$\mathrm{HF}^{20}$; and, 1.25 for COPD. ${ }^{19}$ To calculate a total number of rehospitalisations, we multiplied the number of patients in each group by the RRs and by a factor of 1 for one rehospitalisation, and a factor of 2.5 for two or more rehospitalisations. The total cost of rehospitalisations was obtained by multiplying the number of rehospitalisations by the mean diagnosis-specific hospital admission costs in 2009, as per the Ontario Case Costing Initiative (OCCI). ${ }^{21}$ We calculated the number of hospital-days used in the 1-year period following index hospitalisation for recent quitters and continued smokers by multiplying the total number of rehospitalisations in each group by the mean length of stay for each diagnosis, obtained from OCCI.

\section{Deaths}

We obtained disease-specific mortality rates using published studies of Ontario or Canadian patients. ${ }^{23-25}$ These rates were assigned to patients who had quit smoking. For continued smokers, the mortality rates were multiplied by the following $\mathrm{RR}$ values for death: 1.50 for $\mathrm{AMI}$ or $\mathrm{UA}^{22}$; 1.41 for $\mathrm{HF}^{20}$; and, 1.71 for COPD. ${ }^{19}$

\section{Utility scores and life expectancies}

Utility scores are estimates of health-related quality of life, and are used to calculate the outcome of QALY. Utility values can be between 1 and 0 , with a score of 1 indicating perfect health and 0 representing death. The following utility values used were obtained from a catalogue of scores for chronic conditions in the USA: AMI (0.70), UA (0.69), HF (0.64), and COPD (0.66). ${ }^{26}$ These chronic-condition utilities were then multiplied by utility scores of 0.75 for continued smokers and 0.78 for recent quitters. ${ }^{27}$ Life expectancies for continued smokers and quitters were obtained from published studies, ${ }^{28-30}$ and were discounted by $5 \%$ in the base case, as recommended by Canadian guidelines for the economic evaluation of health technologies. ${ }^{32}$

\section{Usual care costs}

The estimated staff time involved with recording smoking status and providing a patient education booklet is $1 \mathrm{~min}$ at a cost of \$C0.69 per smoker. Currently in Canada, the most widely used patient education booklets are provided through the Canadian Cancer Society (http://www.cancer.ca) and are available at no charge to hospitals. The personnel costs for usual care and intervention were based on the average 2009 hourly rate of a midlevel registered nurse of $\$ C 33.28^{33}$ plus $24 \%$ extended benefits $^{34}$ for a total of \$C41.27.

Table 2 Projected cost outcomes of OMSC intervention compared to usual care in patients with AMI, UA, HF and COPD (\$ in \$C)

\begin{tabular}{|c|c|c|c|}
\hline Cost & $\begin{array}{l}\text { Usual care } \\
(n=15326)\end{array}$ & $\begin{array}{l}\text { OMSC } \\
(n=15326)\end{array}$ & $\begin{array}{l}\text { Costs (savings) } \\
\text { from intervention }\end{array}$ \\
\hline Intervention costs & $\$ 10575$ & $\$ 1139070$ & $\$ 1128495$ \\
\hline \multicolumn{4}{|c|}{ Repeat hospitalisation costs in year following index hospitalisation } \\
\hline $\mathrm{AMI}$ & $\$ 5984453$ & $\$ 5808343$ & $(\$ 176110)$ \\
\hline UA & $\$ 1474391$ & $\$ 1433128$ & (\$41 263) \\
\hline HF & $\$ 10991834$ & $\$ 10777828$ & $(\$ 214007)$ \\
\hline COPD & $\$ 26901393$ & $\$ 26289058$ & $(\$ 612335)$ \\
\hline Total & $\$ 45362645$ & $\$ 45447426$ & $\$ 84781$ \\
\hline Per patient & $\$ 2960$ & $\$ 2965$ & $\$ 5$ \\
\hline
\end{tabular}

AMI, acute myocardial infarction; COPD, chronic obstructive pulmonary diseases; $\mathrm{HF}$, heart failure; OMSC, Ottawa model for smoking cessation; UA, unstable angina pectoris.
Intervention costs

Intervention costs did not include costs related to implementing the programme, but included costs incurred by the hospitals for operating the programme. These costs were based on previous evaluations of the OMSC, and included: (1) personnel costs associated with providing $30 \mathrm{~min}$ of bedside intervention (\$C20.63 per patient); (2) the average daily cost of NRT (\$C2.15 per patient, as determined by the University of Ottawa Heart Institute Department of Pharmacy) multiplied by the relative length of stay for each diagnosis (determined by OCCI); (3) telephone follow-up system and programme database management fees (\$C10.30 per patient; TelASK Technologies, Ottawa, Ontario, Canada); and, (4) personnel costs associated with 40 min of monitoring of the follow-up system (\$C27.24 per patient).

\section{Base case outcomes}

Our base case analysis produced the following intermediate outcomes for usual care and OMSC groups: number of quitters, number of rehospitalisations, number of hospital-days, number of deaths, number of life-years, and number of QALYs. The net cost (total rehospitalisation costs saved minus total intervention costs) and intermediate outcomes were used to calculate the cost-effectiveness ratios. Our primary short-term outcome was 1-year cost per QALY gained. Additional 1-year outcomes were: cost per quitter, cost per hospital-day avoided, and cost per death avoided.

Long-term (ie, over the patient's lifetime) outcomes included lifetime cost per QALY gained and cost per life-year gained (LYG). Mean age and life expectancies were used to calculate total life-years for usual care and OMSC groups. Mean age by diagnosis was obtained from the OMSC database. For long-term QALYs, we multiplied the average life expectancy in each group by the corresponding utility scores.

\section{Sensitivity analyses}

\section{Univariate sensitivity analysis}

We conducted a series of interviews with hospital decision makers $(n=22)$ and provincial healthcare policy analysts $(n=5)$ to determine what additional intervention components to consider in our sensitivity analysis. The most common component of interest was the addition of 12 weeks of cost-free NRT for patients once they are discharged from hospital. Based on recent reviews evaluating the efficacy of population-based and hospitalbased interventions that include NRT, we estimated that the addition of 12 weeks of NRT would increase our programme effectiveness by a relative $58 \%$. $^{3} 35$

For long-term outcomes, costs and benefits were discounted at $5 \%$ in the base case. We used rates of $0 \%$ and $3 \%$ in the sensitivity analysis as recommended by Canadian guidelines for the economic evaluation of health technologies. ${ }^{32}$

\section{Probabilistic sensitivity analysis}

We performed a probabilistic sensitivity analysis to generate uncertainty in the model using Monte Carlo simulation technique for our primary short-term outcome of 1-year cost per QALY gained as well as for lifetime cost per QALY gained. This is a common method used when economic evaluations are based on patient-level or observational data to handle the fact that data elements or 'input parameters' that go into the model are imprecisely estimated from sampled data. In our case, this applies to our input parameters of costs, quit rates, rehospitalisation rates, length of stay values and death rates. Each of these 
parameters included a SD or 95\% CI which were the distributions used to handle the uncertainty in our model. The uncertainty of all the parameters was assessed simultaneously and was characterised as b distributions (probability between 0 and 1). Our model was evaluated 5000 times, with each simulation involving a random draw from each of the input parameter distributions. In our study, 5000 estimates of costs and QALYs were obtained and were presented by two cost-effectiveness acceptability curves (CEAC) - one for short-term and one for long-term QALYs. The CEACs report the probability that the intervention is cost effective compared to usual care for alternative values of a QALY. ${ }^{8}$

\section{RESULTS}

\section{Base case analysis}

Short-term

Table 2 presents the cost outcomes of the OMSC intervention compared to usual care by diagnosis. From the hospital payer perspective, providing the OMSC programme to 15326 smokers with AMI, UA, HF, or COPD would cost an average of \$74 per smoker, or \$1 139070 per year. This investment would generate 4689 new quitters and would prevent 116 repeat hospitalisations, 923 hospital days, and 119 deaths in the first year. The base case intermediate outcomes are presented in table 3 .

Overall, the intervention resulted in the following 1-year costeffectiveness ratios: cost per QALY gained of \$C1386, cost per quitter of \$C20, cost per hospital day avoided of \$C103, and cost per death avoided of \$C803. The disease-specific and overall cost-effectiveness ratios are summarised in table 4 .

\section{Long term}

The OMSC intervention resulted in an overall lifetime cost per QALY gained of \$C61 and cost per LYG of \$C36. The OMSC was dominant (ie, overall costs are lower and QALYs and lifeyears are higher) over usual care in patients with COPD and resulted in greater lifetime QALY and life-years for each of the diagnoses. The lifetime QALY gained were 0.31 per smoker with AMI, 0.29 per smoker with UA, 0.15 per smoker with HF, and 0.20 per smoker with COPD. The overall number of lifeyears gained per smoker was 0.40 .

\section{Sensitivity analyses}

The univariate sensitivity analysis results are presented in table 4 . In the base case analysis, quit smoking medications were only provided to the patients during their hospital admission. The provision of 12 weeks of NRT to patients following their hospitalisation increased the per-patient intervention cost by \$C346, and produced an overall 1-year cost per QALY gained of \$C24 606, and lifetime cost per QALY gained of \$C1071

For the long-term cost-effectiveness ratios, the programme remained cost effective when $0 \%$ and $3 \%$ discounting was applied.

Figure 2 presents the CEAC for 1-year QALY by diagnosis. The probability that the OMSC is most cost effective compared to usual care was greater than $90 \%$ for AMI and HF and over $70 \%$ for UA and COPD for all values of 1-year QALY from \$C0 to \$C100 000 (figure 2).

In the case of lifetime QALY, the CEACs in figure 3 demonstrate that the probability that the OMSC is the most costeffective alternative was greater than $90 \%$ for all diagnoses.

\section{DISCUSSION}

Not surprisingly, our results indicate the economic burden to the hospital system of continued smoking is large. Hospitalisation is an opportune time to initiate smoking cessation treatment as
Table 3 Base case outcomes of OMSC compared to usual care in 2009 cohort of patients with AMI, UA, HF and COPD

\begin{tabular}{|c|c|c|c|}
\hline Outcome & $\begin{array}{l}\text { Usual care } \\
(n=15326)\end{array}$ & $\begin{array}{l}\text { OMSC } \\
(n=15326)\end{array}$ & $\begin{array}{l}\text { Outcomes prevented } \\
\text { (gained) by } \\
\text { intervention }\end{array}$ \\
\hline $\begin{array}{l}\text { Patients who } \\
\text { continue to } \\
\text { smoke, } n\end{array}$ & 12657 & 10637 & 2020 \\
\hline \multicolumn{4}{|c|}{1 year repeat hospitalisations, $\mathrm{n}$} \\
\hline AMI & 626 & 608 & \\
\hline UA & 248 & 241 & \\
\hline $\mathrm{HF}$ & 1096 & 1074 & \\
\hline COPD & 3054 & 2985 & \\
\hline Total & 5024 & 4908 & 116 \\
\hline \multicolumn{4}{|c|}{1 year hospital days, $\mathrm{n}$} \\
\hline $\mathrm{AMI}$ & 3506 & 3403 & \\
\hline UA & 1016 & 988 & \\
\hline $\mathrm{HF}$ & 10631 & 10424 & \\
\hline COPD & 25655 & 25071 & \\
\hline Total & 40808 & 39886 & 923 \\
\hline \multicolumn{4}{|c|}{1 year mortality, $n$} \\
\hline AMI & 687 & 644 & \\
\hline UA & 124 & 117 & \\
\hline $\mathrm{HF}$ & 1197 & 1156 & \\
\hline COPD & 541 & 515 & \\
\hline Total & 2550 & 2431 & 119 \\
\hline \multicolumn{4}{|l|}{ Life years, $\mathrm{n}$} \\
\hline AMI & 40703 & 41701 & \\
\hline UA & 9975 & 10282 & \\
\hline $\mathrm{HF}$ & 8616 & 9015 & \\
\hline COPD & 42497 & 43479 & \\
\hline Total & 101791 & 104476 & (2685) \\
\hline \multicolumn{4}{|c|}{ QALYs (1 year), $n$} \\
\hline AMI & 2584 & 2612 & \\
\hline UA & 716 & 722 & \\
\hline HF & 1059 & 1074 & \\
\hline COPD & 2816 & 2835 & \\
\hline Total & 7175 & 7244 & (69) \\
\hline \multicolumn{4}{|c|}{ QALYs (lifetime), n } \\
\hline AMI & 21701 & 22383 & \\
\hline UA & 5741 & 5920 & \\
\hline $\mathrm{HF}$ & 7189 & 7353 & \\
\hline COPD & 23386 & 23911 & \\
\hline Total & 58017 & 59568 & (1551) \\
\hline
\end{tabular}

AMI, acute myocardial infarction; COPD, chronic obstructive pulmonary diseases; HF, heart failure; OMSC, Ottawa model for smoking cessation; QALY, quality adjusted life year; UA, unstable angina pectoris.

smokers are frequent and high cost users of the healthcare system and are often highly motivated to quit smoking during their admission. Smokers would benefit significantly from receiving cessation support while in hospital in terms of being able to quit smoking and reducing their risk of morbidity and mortality. An estimated \$C547 million is spent each year in Ontario on hospitalisations for AMI, UA, HF and COPD alone. Our study estimates that provision of the OMSC intervention to smokers admitted with these issues would represent $0.20 \%$ of this cost, yet could result in several individual and health system benefits and, in the case of COPD patients, could result in actual cost savings.

However, there is an apparent expectation of behavioural or preventative interventions, including smoking cessation, to demonstrate that they are cost saving or able to save lives with little 
Table 4 Cost-effectiveness ratios of OMSC compared to usual care in 2009 cohort of patients with AMI, UA, HF and COPD (\$ in \$C)

\begin{tabular}{|c|c|c|c|c|}
\hline & \multirow[b]{2}{*}{$\begin{array}{l}\text { Base } \\
\text { case }\end{array}$} & \multicolumn{3}{|c|}{ Univariate sensitivity analyses } \\
\hline & & $\begin{array}{l}12 \text { weeks of } \\
\text { additional } \\
\text { pharmacotherapy }\end{array}$ & $\begin{array}{l}\text { Discount } \\
\text { rate of } 0 \%\end{array}$ & $\begin{array}{l}\text { Discount } \\
\text { rate of } 3 \%\end{array}$ \\
\hline \multicolumn{5}{|c|}{ Short-term outcomes } \\
\hline \multicolumn{5}{|c|}{1 year cost per QALY gained } \\
\hline AMI & $\$ 6874$ & $\$ 25496$ & & \\
\hline UA & $\$ 8482$ & $\$ 32328$ & & \\
\hline $\mathrm{HF}$ & $\$ 472$ & $\$ 19834$ & & \\
\hline COPD & Dominant & $\$ 24126$ & & \\
\hline Overall & $\$ 1386$ & $\$ 24606$ & & \\
\hline \multicolumn{5}{|c|}{ Cost per quitter } \\
\hline AMI & $\$ 99$ & $\$ 552$ & & \\
\hline UA & $\$ 107$ & $\$ 608$ & & \\
\hline $\mathrm{HF}$ & $\$ 9$ & $\$ 523$ & & \\
\hline COPD & Dominant & $\$ 469$ & & \\
\hline Overall & $\$ 20$ & $\$ 528$ & & \\
\hline \multicolumn{5}{|c|}{ Cost per hospital-day avoided } \\
\hline AMI & $\$ 1893$ & $\$ 7020$ & & \\
\hline UA & $\$ 1904$ & $\$ 7259$ & & \\
\hline $\mathrm{HF}$ & $\$ 34$ & $\$ 1244$ & & \\
\hline COPD & Dominant & $\$ 796$ & & \\
\hline Overall & $\$ 103$ & $\$ 1808$ & & \\
\hline \multicolumn{5}{|c|}{ Cost per death avoided } \\
\hline AMI & $\$ 4494$ & $\$ 16668$ & & \\
\hline UA & $\$ 7790$ & $\$ 27784$ & & \\
\hline HF & $\$ 169$ & $\$ 7544$ & & \\
\hline COPD & Dominant & $\$ 17435$ & & \\
\hline Overall & $\$ 803$ & $\$ 14658$ & & \\
\hline \multicolumn{5}{|c|}{ Long-term outcomes } \\
\hline \multicolumn{5}{|c|}{ Lifetime cost per QALY gained } \\
\hline AMI & $\$ 286$ & $\$ 1062$ & $\$ 109$ & $\$ 207$ \\
\hline UA & $\$ 303$ & $\$ 1154$ & $\$ 118$ & $\$ 220$ \\
\hline $\mathrm{HF}$ & $\$ 42$ & $\$ 1564$ & $\$ 23$ & $\$ 34$ \\
\hline COPD & Dominant & $\$ 885$ & Dominant & Dominant \\
\hline Overall & $\$ 68$ & $\$ 1071$ & $\$ 24$ & $\$ 45$ \\
\hline \multicolumn{5}{|c|}{ Cost per LYG } \\
\hline AMI & $\$ 196$ & $\$ 726$ & $\$ 68$ & $\$ 137$ \\
\hline UA & $\$ 177$ & $\$ 674$ & $\$ 69$ & $\$ 128$ \\
\hline $\mathrm{HF}$ & $\$ 17$ & $\$ 723$ & $\$ 11$ & $\$ 15$ \\
\hline COPD & Dominant & $\$ 474$ & Dominant & Dominant \\
\hline Overall & $\$ 36$ & $\$ 629$ & $\$ 14$ & $\$ 26$ \\
\hline
\end{tabular}

investment. By contrast, this requirement does not exist to nearly the same extent when it comes to other medical or procedural interventions for chronic diseases, for instance, treatments for hyperlipidaemia or vascular surgeries. Given the known benefits of smoking cessation on morbidity and mortality, it is our opinion that cessation interventions should be adopted regardless of their ability to save money. If smoking cessation interventions are indeed cost saving, this should be seen simply as a favourable consequence of helping people to quit.

\section{Results as compared to other studies}

An earlier investigation revealed that a nurse-led smoking cessation intervention for patients hospitalised for AMI cost \$C220

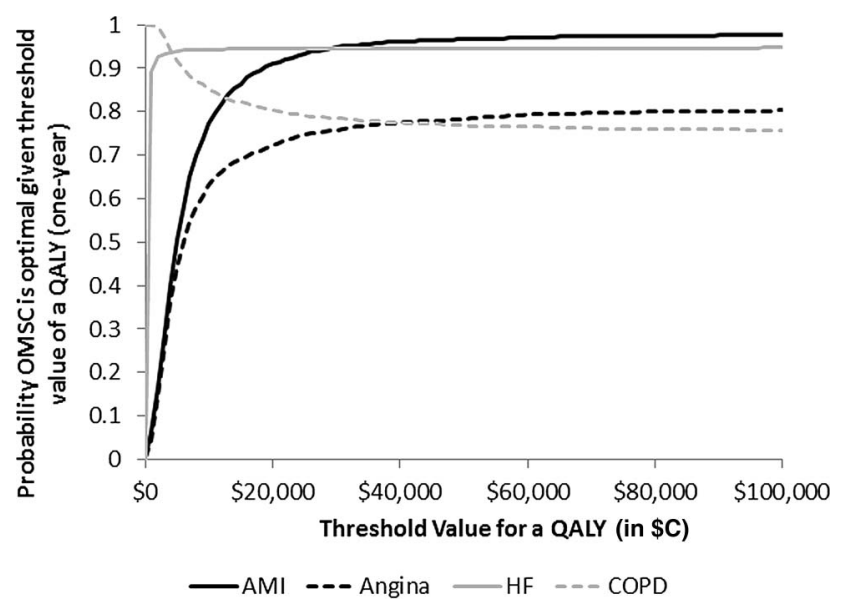

Figure 2 Cost effectiveness acceptability curves by disease showing change in the probability that the OMSC is cost-effective as the value of QALY (1-year) changes. OMSC, Ottawa model for smoking cessation. QALY, quality adjusted life year;

per LYG in 1991 US dollars (approximately \$C628 per LYG in current Canadian dollars). ${ }^{36}$ In that study, the intervention was estimated to reduce smoking by $26 \%$, more than twice as much as our estimate. We believe our estimate of the effect of the OMSC intervention on quitting is a more accurate reflection of what can be achieved in 2012. More recently, Ladapo et $a l^{6}$ projected the cost-effectiveness of smoking cessation interventions for a hypothetical US cohort of 327600 smokers hospitalised with AMI employing in-hospital counselling and follow-up telephone calls from a nurse after discharge, but did not include the use of pharmacotherapy in the base case; their programme would cost \$C540 per quitter and \$C4350 per LYG. Comparatively, the OMSC cost-effectiveness ratios for smokers with AMI of \$C99 per quitter and \$C196 per LYG can be considered highly appealing.

Our long-term cost-effectiveness ratios are also highly attractive when compared to several other interventions routinely used to treat patients with smoking-related conditions (or their sequelae), including: b-blocker use after AMI $(\approx \$ C 10000 \text { per LYG })^{37}$; inhaled corticosteroids for treatment of COPD $(\approx \$ C 30000$ per

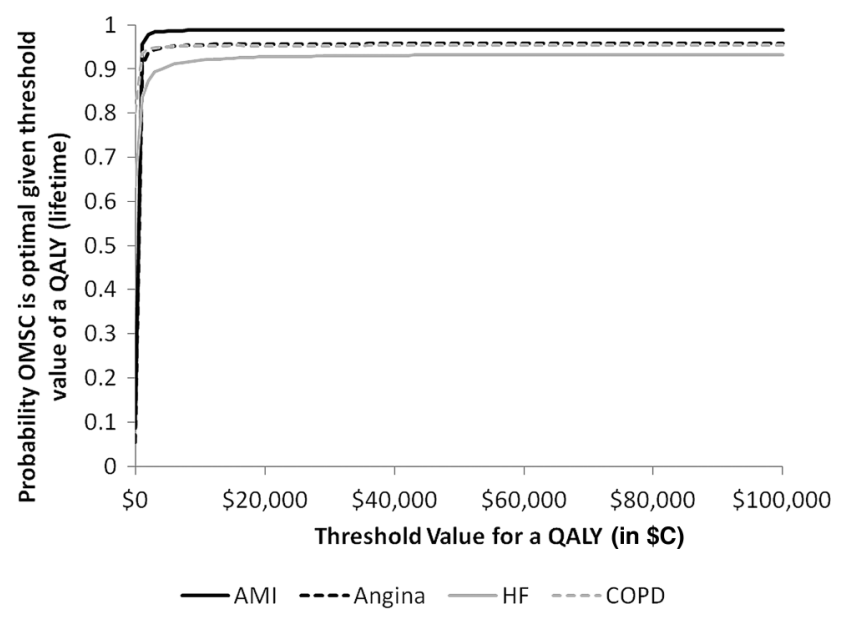

Figure 3 Cost effectiveness acceptability curves by disease showing change in the probability that the OMSC is cost-effective as the value of QALY (lifetime) changes. OMSC, Ottawa model for smoking cessation. QALY, quality adjusted life year; 
LYG $)^{38}$; cardiac resynchronisation therapy for HF $(\approx \$ C 50000$ per LYG $)^{39}$; hemodialysis for renal insufficiency $(\approx \$ C 50000$ per $\mathrm{LYG})^{40}$; and, percutaneous coronary intervention for coronary artery stenosis (>\$C200 000 per LYG) ${ }^{41}$

\section{Study limitations}

Our study has several limitations. We did not model dynamic changes in smoking status that can occur over time, nor did we have data concerning effects of the OMSC on quit rates longer than 12 months after hospitalisation. Only a small percentage of smokers (3-5\%), however, spontaneously quit smoking, and smokers who are abstinent at 12 months have a 95\% likelihood of being smoke-free at 24-month follow-up. ${ }^{42}$

We did not account for smoking cessation interventions that may have occurred outside of the hospital in either group. It is possible that smokers may have received support that would have generated additional costs; however, we did not model these potential costs in our current study. Before applying our results, readers need to consider that four distinct patient populations were examined in our model, and results may not be transferable to other patient populations. We examined a moderately intensive intervention provided in an acute care setting, and application of our data to other interventions or healthcare settings should be done with caution. Further, usual care or the most common alternatives available in other countries may differ from the usual care scenario in Canada that was applied in our model.

Some of our data elements, for instance, our RR of rehospitalisation and death and our utility score estimates, were drawn from older studies, and in the case of COPD risks, a longitudinal observational trial. ${ }^{19} 20222730$ We included distributions for all input parameters except utility scores and life expectancies as they were unavailable in the studies we used; therefore, we were unable to assess the uncertainty of these parameters in our probabilistic sensitivity analysis.

It might be speculated that patients who quit smoking after hospitalisation are sicker than those who continue to smoke. Because our risk estimates were drawn from observational data, they likely underestimate the harms of continued smoking. We did not consider the effects of quitting smoking on preventing other important smoking-attributable illnesses (eg, cancers, stroke, peripheral vascular disease, other respiratory diseases, and other cardiac diseases). We chose to examine costs and outcomes specifically related to hospitalisations. There is a dearth of information when it comes to the impact of smoking cessation on other important healthcare system costs and savings, for instance, those related to emergency department, specialty-care and primary-care visits. Future studies should examine a wider array of healthcare impacts, and consider a societal perspective that would also take into account productivity gains or losses. Quitting smoking has been associated with gains in societal productivity; it is thought that the inclusion of such information would further improve an understanding of the costeffectiveness of the intervention. ${ }^{43}{ }^{44}$ Our study only examined costs and benefits that occurred in the first year. Evidence suggests that patients who quit may be at reduced risk for hospitalisation, morbidity and death in subsequent years, as the benefits of cessation accrue over time. ${ }^{72329} 30$

\section{CONCLUSIONS}

Due to population ageing and the costly burden of chronic diseases that exists globally, hospitals and healthcare payers are searching for cost-effective solutions, including primary and secondary prevention strategies. Implementation of the OMSC hospital-initiated intervention for smoker-patients appears to be a highly cost-effective option from the hospital payer's perspective. Important consideration is the relatively low intervention cost compared to the reduction in costs related to readmissions for illnesses associated with continued smoking.

\section{What this paper adds}

- Most hospitals fail to offer tobacco cessation interventions due, in part, to concerns about the perceived costs of such programs. Cost-effectiveness analyses provide health care administrators with information needed to guide the allocation of resources.

- This study demonstrated that hospital-initiated smoking cessation interventions, like the Ottawa Model for Smoking Cessation, can be implemented with relatively small investment and can be extremely cost-effective when applied to high risk smokers with existing chronic disease.

- Given the known benefits of smoking cessation on morbidity and mortality, cessation interventions should be adopted by hospitals regardless of their ability to save money. If these interventions result in cost-savings, this should be seen simply as favourable consequence of helping people to quit.

Acknowledgements The authors would like to acknowledge the contributions of Laura Jones of the University of Ottawa Heart Institute for her assistance in data gathering and management. In addition, we would like to thank the peer-reviewers for their thoughtful suggestions which, we feel, have enhanced the current version.

Contributors RDR and KAM conceived the study. KAM, HVN and BP compiled the sources and developed and analysed the economic model with the guidance of DC. $D C$ advised and reviewed the data and completed the sensitivity analyses. KAM and RDR drafted the manuscript. KAM, DC, DM, HVN, BP, ALP and RDR contributed to analysis and outcome planning and to the writing, reviewing, and editing of the final manuscript. KAM is the guarantor.

Funding This project was supported by a Contribution Agreement between the University of Ottawa Heart Institute and the Ontario Ministry of Health and Long Term Care. All authors declare independence from the funder. The funders had no role in study design, data collection and analysis, decision to publish, or preparation of the manuscript.

Competing interests ALP has served as a consultant to Pfizer, GlaxoSmithKline, Johnson \& Johnson and Amgen; and, received educational and research support from Pfizer \& Johnson \& Johnson. RDR has received research support, speaker and consultation fees from Pfizer.

Provenance and peer review Not commissioned; externally peer reviewed.

Data sharing statement Unpublished data from the study, specifically deidentified and economic model data, can be made available to editors and reviewers upon request to corresponding author.

Open Access This is an Open Access article distributed in accordance with the Creative Commons Attribution Non Commercial (CC BY-NC 3.0) license, which permits others to distribute, remix, adapt, build upon this work non-commercially, and license their derivative works on different terms, provided the original work is properly cited and the use is non-commercial. See: http://creativecommons.org/ licenses/by-nc/3.0/

\section{REFERENCES}

1 World Health Organization. Economics of tobacco toolkit: assessment of the economic costs of smoking. Geneva, Switzerland: World Health Organization Press, 2011.

2 U.S. Department of Health and Human Services. The health consequences of smoking: a report of the surgeon general. Atlanta, GA: Centers for Disease Control and Prevention, National Center for Chronic Disease Prevention and Health Promotion, Office on Smoking and Health, 2004.

3 Rigotti NA, Clair C, Munafo MR, et al. Interventions for smoking cessation in hospitalised patients. Cochrane Database Syst Rev 2012;(5):CD001837. 
4 Reid RD, Pipe AL, Quinlan B. Promoting smoking cessation during hospitalization for coronary artery disease. Can J Cardiol 2006;22:775-80.

5 Reid RD, Mullen KA, Slovinec D'Angelo ME, et al. Smoking cessation for hospitalized smokers: an evaluation of the "Ottawa Model". Nicotine Tob Res 2010;12:11-18.

6 Ladapo JA, Jaffer FA, Weinstein MC, et al. Projected cost-effectiveness of smoking cessation interventions in patients hospitalized with myocardial infarction. Arch Intern Med 2011;171:39-45.

7 Mohiuddin SM, Mooss AN, Hunter CB, et al. Intensive smoking cessation intervention reduces mortality in high-risk smokers with cardiovascular disease. Chest 2007;131:446-52.

8 Drummond MF, Sculpher MJ, Torrance GW, et al. Methods for the evaluation of health care programmes. 3rd edn. New York, NY: Oxford University Press, 2005.

9 Kind $\mathrm{P}$, Lafata JE, Matuszewski $\mathrm{K}$, et al. The use of QALYs in clinical and patient decision-making: issues and prospects. Value Health 2009;12:S27-30.

10 Reid JL, Hammond D, Burkhalter R, et al. Tobacco Use in Canada: Patterns and Trends. 2012. http://www.tobaccoreport.ca (accessed Oct 2013).

11 Pipe AL, Eisenberg MJ, Gupta A, et al. Smoking cessation and the cardiovascular specialist: Canadian Cardiovascular Society position paper. Can J Cardiol 2011;27:132-7.

12 Canadian Institute for Health Information. Highlights of 2009-2010 Inpatient Hospitalizations and Emergency Department Visits. 2011. https://secure.cihi.ca/ estore/productSeries.htm?pc=PCC526 (accessed Oct 2013).

13 Canadian Institute for Health Information. Total number of acute inpatient hospitalizations for each of the five conditions as the MRDX, in Ontario, by fiscal year. Data provided by Division Support Services, 2012.

14 Reid RD, Pipe AL, Quinlan B, et al. Interactive voice response telephony to promote smoking cessation in patients with heart disease: a pilot study. Patient Educ Couns 2007;66:319-26

15 Registered Nurses' Association of Ontario. Integrating Smoking Cessation into Daily Nursing Practice. Best Practice Guidelines 2007. http://www.rnao.org/bestpractices (accessed Mar 2014).

16 Reid RD; On behalf of CAN-ADAPTT's Clinical Practice Guideline Development Group. Hospital-based Populations CAN-ADAPTT_Canadian Smoking Cessation Guideline 2011. http://www.nicotinedependenceclinic.com/English/CANADAPTT/ Guideline/Hospital\%20Based\%20Populations/Home.aspx (accessed Mar 2014)

17 Canadian Institute for Health Information. Health indicators 2008. Ottawa, ON: $\mathrm{CIHI}, 2008$

18 Canadian Institute for Health Information. Health ilndicators 2009. Ottawa, ON: $\mathrm{CIHI}, 2009$.

19 Godtfredsen NS, Vestbo J, Osler M, et al. Risk of hospital admission for COPD following smoking cessation and reduction: a Danish population study. Thorax 2002:57:967-72.

20 Suskin N, Sheth T, Negassa A, et al. Relationship of current and past smoking to mortality and morbidity in patients with left ventricular dysfunction. J Am Coll Cardiol 2001:37:1677-82.

21 Ontario Case Costing Initiative (OCCI). Costing Analysis (CAT) Tool. http://www. occp.com/mainPage.htm (accessed Oct 2013).

22 Critchley JA, Capewell S. Mortality risk reduction associated with smoking cessation in patients with coronary heart disease: a systematic review. JAMA 2003:290:86-97.

23 Gershon AS, Wang C, Wilton AS, et al. Trends in chronic obstructive pulmonary disease prevalence, incidence, and mortality in Ontario, Canada, 1996 to 2007: a population-based study. Arch Intern Med 2010;170:560-65.
24 Yan AT, Tan M, Fitchett D, et al. One-year outcome of patients after acute coronary syndromes (from the Canadian Acute Coronary Syndromes Registry). Am J Cardiol 2004;94:25-9.

25 Yeung DF, Boom NK, Guo H, et al. Trends in the incidence and outcomes of heart failure in Ontario, Canada: 1997 to 2007. CMAJ 2012;184:E765-73.

26 Sullivan PW, Ghushchyan V. Preference-Based EQ-5D index scores for chronic conditions in the United States. Med Decis Making 2006;26:410-20.

27 Tillmann M, Silcock J. A comparison of smokers' and ex-smokers' health-related quality of life. J Public Health Med 1997;19:268-73.

28 Alter DA, Ko DT, Tu JV, et al. The average lifespan of patients discharged from hospital with heart failure. J Gen Intern Med 2012;27:1171-9.

29 Shavelle RM, Paculdo DR, Kush SJ, et al. Life expectancy and years of life lost in chronic obstructive pulmonary disease: findings from the NHANES III Follow-up Study. Int J Chron Obstruct Pulmon Dis 2009;4:137-48.

30 Taylor DH Jr, Hasselblad V, Henley SJ, et al. Benefits of smoking cessation for longevity. Am J Public Health 2002;92:990-6.

31 Hughes JR, Peters EN, Naud S. Relapse to smoking after 1 year of abstinence: a meta-analysis. Addict Behav 2008;33:1516-20.

32 Canadian Agency for Drugs and Technologies in Health (CADTH). Guidelines for the economic evaluation of health technologies: Canada. 3rd edn. Ottawa, ON: CADTH, 2006.

33 Ontario Nurses' Association. ONA-Frequently Asked Questions—Salary. http:// www.ona.org/faqs.html\#f16 (accessed Sep 2013).

34 Mercer Canada Limited (Commisioned by the Ontario Chamber of Commerce). Cost Trends in Health Benefits for Ontario Businesses: Analysis for Discussion. 2011. http://www.occ.ca/Publications/Cost-Trends-in-Health-Benefits-Report.pdf (accessed Feb 2014).

35 Stead LF, Perera R, Bullen C, et al. Nicotine replacement therapy for smoking cessation. Cochrane Database Syst Rev 2012;11:CD000146.

36 Krumholz HM, Cohen BJ, Tsevat J, et al. Cost-effectiveness of a smoking cessation program after myocardial infarction. J Am Coll Cardiol 1993;22:1697-702.

37 Levy AR, Briggs AH, Demers $C$, et al. Cost-effectiveness of beta-blocker therapy with metoprolol or with carvedilol for treatment of heart failure in Canada. Am Heart J 2001;142:537-43.

38 Rutten-van Molken M, Lee TA. Economic modeling in chronic obstructive pulmonary disease. Proc Am Thorac Soc 2006;3:630-4.

39 Sanders GD, Hlatky MA, Owens DK. Cost-effectiveness of implantable cardioverter-defibrillators. N Engl J Med 2005;353:1471-80.

40 Winkelmayer WC, Weinstein MC, Mittleman MA, et al. Health economic evaluations: the special case of end-stage renal disease treatment. Med Decis Making 2002;22:417-30.

41 Weintraub WS, Boden WE, Zhang Z, et al. Cost-effectiveness of percutaneous coronary intervention in optimally treated stable coronary patients. Circ Cardiovasc Qual Outcomes 2008;1:12-20.

42 Gilpin EA, Pierce JP, Farkas AJ. Duration of smoking abstinence and success in quitting. J Natl Cancer Inst 1997;89:572-6.

43 Halpern MT, Shikiar R, Rentz AM, et al. Impact of smoking status on workplace absenteeism and productivity. Tob Control 2001;10:233-8.

44 Kahende JW, Loomis BR, Adhikari B, et al. A review of economic evaluations of tobacco control programs. Int J Environ Res Public Health 2009:6:51-68 